\title{
Moderating role of cannabis use between insight and depression in early psychosis
}

Julien Elowe ${ }^{a, b}$, Philippe Golayc ${ }^{c}$ Philipp S. Baumann ${ }^{d}$, Alessandra Solida-Tozzid, Philippe Conus ${ }^{d}$

a Corresponding author

b Department of Psychiatry, Lausanne University Hospital, Prangins Psychiatric Hospital (CHUV), Chemin Oscar Forel 3, 1196 Prangins, Switzerland

c Community Psychiatry Service, Department of Psychiatry, Lausanne University Hospital, Lausanne, Switzerland

d Service of General Psychiatry, Treatment and Early Intervention in Psychosis Program (TIPP-

Lausanne), Lausanne University Hospital (CHUV), Hôpital de Cery, 1008 Prilly, Switzerland

Julien Elowe

Hôpital Psychiatrique de Prangins

Chemin Oscar Forel 3

$\mathrm{CH}-1197$ Prangins

Phone : +41213142317

Fax : +41213148810

julien.elowe@chuv.ch

Word counts

Abstract 249

Text body 2'965 


\section{Introduction}

In spite of its conceptual complexity and the clinical challenges it incurs, insight is recognized as a central element in the evaluation process of first episode psychosis (FEP) and a key determinant of its treatment. Indeed it has been shown to be positively correlated to important prognostic factors such as medication adherence (Fenton et al., 1997, Olfson et al., 1999) and functional outcome (Saravanan et al., 2010). On a clinical level, the variability of results around a possible relationship between insight and symptom intensity has been suggested that insight might be influenced by a combination of "trait" and "state" factors (for a complete review of insight, refer to (Elowe and Conus, 2017)). However, while insight can have a positive impact on FEP, it may also be associated with increased depression level (Misdrahi et al., 2014) and suicidal behavior (Vilaplana et al., 2015), phenomenon coined as the "insight paradox" which has been confirmed by a recent meta-analysis (Belvederi Murri et al., 2015). More specifically, higher levels of the self-reflection component of cognitive insight showed a significant positive correlation with depression (Palmer et al., 2015). Several studies have tried to identify factors that could moderate this association such as the patients' beliefs about the illness, including the contingency of self-stigma (Lysaker et al., 2007), their coping strategies and their struggle for recovery (Belvederi Murri et al., 2015), premorbid adjustment (Campos et al., 2011) or deficits in social cognition and metacognition (Lysaker et al., 2013). Assessment of neurocognitive function and phenomenological reflection have also started to attract attention as potential confounding variables of the relationship between insight and depression (Belvederi Murri et al., 2015) as well as the concept of social identity (Klaas et al., 2017).

Surprisingly, no study has questioned the role of cannabis use as a potential mediator in the link between insight and depression. This is astonishing, considering 
the high prevalence of its use among early psychosis patients (Zammit et al., 2008) and its impact on various aspects of the disorder. Indeed, besides increasing the risk to develop psychosis if used in high quantity at an early age (Lambert et al., 2005), patients who use cannabis have been found to have a poorer functional outcome than patients who do not (Zammit et al., 2008). Moreover, cannabis users seem to be more prevalent among FEP patients who are non-adherent to medication compared with adherent FEP patients (Coldham et al., 2002).

Interestingly, a recent meta-analysis has brought strong arguments for an association between cannabis use and a higher risk to develop depressive disorders (Lev-Ran et al., 2014). While the authors recognize the possible implication of significant confounding or common factors, there is no mention of the possible moderating role of insight in this association. This issue is of importance considering that if proven true, it may provide a new treatment target for the prevention of depression in FEP. With this in mind we explored, in a cohort of FEP patients followed-up over 36 months, if patients with high insight and using more cannabis at the beginning of the program would display more depressive symptoms after the first year after entry into the program. The first aim of our prospective study was to examine if cannabis use moderates the alleged association between baseline insight and depression one year later. Because adequate medication could play an important role in depression levels, medication adherence was included in the models.

Our secondary aim was to explore the evolution of that association between 12 and 36 months after entry into the program, and the potential relationship between baseline predictors such as insight, cannabis use and medication adherence, on positive and negative symptomatology on the one hand, and functional outcome on the other hand. 


\section{Material and methods}

\subsection{Subjects}

Data were gathered among recent-onset FEP patients enrolled in a specialized early psychosis program named TIPP (Treatment and early Intervention in Psychosis Program), designed and launched in 2004 at the Department of Psychiatry in Lausanne (CHUV), Switzerland (Baumann et al., 2013). Patients are referred to the program by the hospital, general practitioners, social professional networks or families, and regardless of their socioeconomic status. Therefore, the study sample is representative of the entire population of patients with first-episode psychosis who need specialized psychiatric treatment. Inclusion criteria to the program are age between 18 and 35, residence in the catchment area around Lausanne (population of about 300'000) and meeting criteria for psychosis according to the "Psychosis threshold" subscale of the Comprehensive Assessment of At Risk Mental State (CAARMS) scale (Yung et al., 2005). Subjects who received antipsychotic medication for a total duration of more than six months, or those with mental retardation (intelligence quotient below 70), or displaying psychosis secondary to substance use or organic brain disorders are referred to other treatment programs.

The Research and Ethics Committee of the Faculty of Biology and Medicine of Lausanne University granted permission to access all clinical data for research purposes, and all patients who enter the program do so on a voluntary basis and are automatically included in the study if they meet the clinical inclusion criteria. After the inclusion, the TIPP program consists of a three-year individual treatment by a psychiatrist and a case manager, antipsychotic medication, psychosocial and family interventions whenever needed. Cannabis users are not provided with a specific intervention apart from psychoeducation about the effects of pursuing cannabis use 
in the context of psychosis. A questionnaire has been designed to assess sociodemographic elements, past medical and psychiatric history, insight into the illness, substance use, adherence to medication, exposure to life events, and global functioning. At baseline, it is completed by the case managers, and additional relevant information is eventually computed during follow-up. Throughout the threeyear program, similar follow-up assessments are made at 2, 6, 12, 18, 24, 30, and 36 months respectively. Symptomatology is assessed by trained psychologists and psychiatrists within the same deadlines. Symptom assessment was conducted by a psychologist who was $100 \%$ independent of patients' treatment and had received standardized training prior to the study. Inter rater reliability standards for the PANSS have been verified throughout the training using video-taped interviews and consensus reference ratings.

\subsection{Definitions and assessments}

The diagnostic procedure starts at the first contact with psychiatric care. All clinical and diagnostic elements available at the entry of the program are taken into consideration and integrated in the diagnostic process that extends throughout the program. The consensus diagnostic procedure is realized by a senior psychiatrist and the senior psychologist in charge of scale based assessment over the treatment period. They both review the entire file once after 18 months and again after 36 months, or at the end of treatment, and conduct a diagnostic process based on DSMIV criteria (American Psychiatric Association, 2000). This final diagnosis was considered in the present study.

Insight was assessed at baseline, 2, 12, 24, and 36 months respectively, by the case managers using a Likert type scale (Conus et al., 2007). Thus, insight was considered either absent (score 0), partial (score 1), or totally present (score 2). This 
rating was negatively correlated ( $\rho$ ranging between -.547 and -.414 ) with the $\mathrm{G} 12$ PANSS item at all follow-up assessments, suggesting adequate convergent validity. Information about adherence to medication is collected from the subject and his family. Patients taking their medication between 75 and $100 \%$ of the time are considered totally adherent to medication. Patients taking their medication between 25 and $75 \%$ of the time are considered partially adherent to medication. Patients taking their medication less than $25 \%$ of the time are considered non adherent to medication.

Symptomatology was assessed by means of a battery of measures at entry into the program, at $2,6,12,18,24,30$, and 36 months after entry into the program. The battery includes the Positive and Negative Syndrome Scale (PANSS), the Brief Psychiatric Rating Scale (BPRS), the Clinical Global Impression (CGI), the Young Mania Rating Scale (YMRS), and the Montgomery-Asberg Depression Rating Scale (MADRS).

Cannabis use was assessed at the same time points with the Case Manager Rating Scale (CMRS) (Drake et al., 1990) which grades level of use on a continuum from absent - 1 - to extremely severe -5 -.

Assessment of global functioning was made with the Social and Occupational Functioning Assessment Scale (SOFAS) (Morosini et al., 2000).

\subsection{Statistical analysis}

In order to study whether the relationship between insight and the different outcomes could be moderated by medication adherence and substance use, a series of multivariate regression models were estimated.

3. Baseline insight, medication adherence and cannabis use score were entered as independent variables while the PANSS positive score, PANSS negative 
score, the MADRS score and the SOFAS score after one year were alternately selected as the dependent variable. Two-way and three way interaction terms between insight, medication adherence and cannabis use were also entered as predictors. In order to avoid multicollinearity issues, predictors were centered before computing the interaction terms. Significance of simple and interaction terms allows to study whether predictors of various outcomes have additive respectively multiplicative effects. In other words, they answer the question whether baseline medication adherence and substance use do in fact moderate the relationship between insight and the outcomes. The various scores were used in a continuous way to estimate the model parameters. To facilitate interpretation, graphs were built with high and low scores corresponding to levels one standard deviation over/below the mean. These regression models were estimated with IBM SPSS version 23. All statistical tests were two-tailed and significance was determined at the .05 level.Results

\subsection{Patient characteristics}

Data stem from the prospective follow-up of the first 214 patients who were enrolled in the program and who completed 36 months of assessment.

The sociodemographic and baseline clinical characteristics of the study sample are given in table 1.

\subsection{Moderating role of cannabis use in the insight - depression association}

There was no main effect of insight, medication adherence and cannabis use at the beginning of the program on depression measured 12 months later. However, the three-way interaction term (cannabis use $\mathrm{x}$ insight $\mathrm{x}$ medication adherence) was significant $(\beta=.405, p=.020)$ revealing a complex relationship between these three variables and depression. The results are depicted in figure 1. High and low levels correspond to scores one standard deviation above respectively below the mean. While a high level of insight tends to be significantly associated with higher MADRS scores in patients with high medication adherence and high cannabis use, 
depression decreases significantly in patients with high medication adherence and low cannabis use when insight is present. Finally, there is almost no effect of insight on depression scores with patients with low medication adherence and heavy cannabis use.

\subsection{Evolution of the relationship between depression and insight over time}

While the level of depression tends to globally decrease over the three-year program, we found no association between any of the independent variables measured at the beginning of the program and depression after 24 and 36 months respectively.

\subsection{Positive and negative symptomatology}

Low cannabis use at baseline negatively predicted positive symptomatology after 12 $(\beta=.248, p=.029)$, and 36 months $(\beta=.272, p=.046)$ respectively.

We found a significant three way interaction term (cannabis use $\mathrm{x}$ insight $\mathrm{x}$ medication adherence) $(\beta=.571, p=.001)$ when predicting positive symptomatology after 24 months. The results are depicted in figure 2. In other words, while a high baseline level of insight tends to be significantly associated with higher PANSS positive scores two years later in patients with high medication adherence and high cannabis use, positive symptomatology decreases significantly in patients with high medication adherence and low cannabis use when insight is present. At the entry into the program, and at 12 and 24 months respectively, insight always predicted negative symptomatology after 24 months of follow-up.

\subsection{Global functioning (SOFAS)}

We found that the level of insight always predicted global functioning throughout the program. In particular, insight at the beginning of the program was associated with higher functioning 12 months later $(\beta=.178, p=.037)$ and 24 months later $(\beta=.189$, 
$\mathrm{p}=.045)$. Other variables like medication adherence and cannabis use were not associated with functioning. Finally, the relationship between insight at the beginning and functioning 3 years later was not significant anymore $(\beta=.132, p=.174)$

\section{Discussion}

While the question of the relationship between depression and insight in psychosis has been examined in many respects and is now recognized, the knowledge is still limited regarding the possible moderators or confounding factors of this link (Belvederi Murri et al., 2015), even more so in the early phase of psychosis. To our knowledge, this is the first prospective study conducted with FEP patients exploring the potential moderating role of cannabis use in the relationship between insight and depression. Such a study is important considering that the identification of moderating factors linking insight and depression may become interesting targets for treatment in order to mitigate the negative consequences of the development of insight.

The main finding of this study is that, among patients presenting with high insight and high medication adherence at the entry into a specialized intervention program, heavy cannabis users at baseline have significantly higher levels of depression after 12 months of treatment compared to low-users. In contrast, we did not observe such a correlation between cannabis use and depression level at 12 months neither in patients with low level of insight at baseline nor in those with poor adherence to medication despite high level of insight. Previous studies have brought arguments that the endocannabinoid system is possibly related to mood, through an increase in serotoninergic and noradrenergic activity (Bambico and Gobbi, 2008). While longitudinal studies on this issue have yielded inconsistent results, most of them have concluded that frequent early-onset cannabis use is linked to a higher risk of later 
depression (Moore et al., 2007). However, confounding or common factors (biological, personality, social and environmental) probably coexist in this association, explaining the discrepancies described above.

Taken together, our results give support to the hypothesis that cannabis may be a moderator in the emergence of depressive symptoms in FEP patients with high level of insight and good adherence to treatment. In other words, among patients with good insight and who are adherent to treatment, those using cannabis are at increased risk to develop depressive symptoms and may therefore need specific attention, considering their increased suicide risk (Coentre et al., 2017). In particular, interventions aiming at reduction of cannabis use should be offered to these patients such as web-based programs (Rooke et al., 2013), comprehensive family-based treatment, counseling and prevention information, or cognitive behavioral therapy (CBT)-oriented motivational psychotherapy (Olmos et al., 2018).

The association between cannabis use and higher level of depressive symptoms in this patient subgroup was not present at 24 and 36 months. This finding was surprising at first glance and caught our attention. One possible explanation could be related to the concept of social identity suggesting that patients with high level of insight go through a phase of depression that resolves when they have been able to build a new sense of identity, which integrates the episode. Furthermore, this positive evolution may also be explained by the intensive and comprehensive nature of the integrated treatment proposed to patients in a setting similar to other specialized programs that have been shown to foster a reduction of depressive symptoms and suicidal behavior over time (Coentre et al., 2017).

We also found that patients with a high cannabis use (in addition to having high baseline level of insight and medication adherence), have significantly higher PANSS positive scores two years later, than patients with low cannabis use at baseline (in 
addition to having a high level of insight and high medication adherence). These results are in line with previous findings (Schimmelmann et al., 2012), showing that those with baseline cannabis use present with higher illness severity.

Several limitations of this study should be taken into consideration. First, and as discussed earlier, insight is a complex concept that might be affected by many variables that we have not taken into consideration such as internalized stigma. While some studies have suggested stigma being a potential moderator acting between insight and depression (Lysaker et al., 2013), others do not support this hypothesis (Grover et al., 2018). Second, insight was assessed using a simple threepoint Likert scale, possibly lacking precision. Others have commented on the complexity of the concept of insight, arguing that it comprises many overlapping constructs. Nonetheless, other Likert-scale type instruments have shown good convergent and discriminant validity and reliability (Gerretsen et al., 2014). Third, data regarding evolution of cannabis use over the treatment period was not taken into consideration, and such fluctuations may have had an influence on depressive symptoms. As described above, the TIPP program does not provide cannabis users with a specific intervention apart from psychoeducation about the effects of pursuing cannabis use in the context of psychosis. Therefore, cannabis use is a variable that has been considered as a continuum and was assessed at the seven time points throughout the three-year program. Moreover, while a decline by approximately $50 \%$ in cannabis use after first episode psychosis is now recognized at follow-up, it usually spreads over a number of years (Myles et al., 2016). Fourth, other factors known to be possibly associated with depression in first episode psychosis have not been taken into account, such as duration of untreated psychosis (DUP). Since the DUP is estimated based on the often inaccurate recall of the patient and his or her family informants, we chose to exclude this variable to simplify the analysis. Finally, data on 
pharmacological therapy, mainly the use of antidepressants in the follow up period was not taken into consideration in our analysis and could affect the prevalence of depression.

In conclusion, while substantial work has demonstrated the high prevalence of depression and suicidal behaviour in first episode psychosis, the present study suggests that cannabis use continuation during the year following a first episode psychosis may play a significant role in the development or the maintenance of postpsychotic depression in patients who present with high level of insight and adherence to medication. In the context of early intervention programs, specific attention should therefore be paid to this subgroup of patients and specific therapeutic strategies should be proposed considering the high risk of suicide in such patients.

The authors have no conflicts of interest to disclose and have not been paid for this article.

The authors thank the subjects whose participation made this study possible.

American Psychiatric Association, 2000. Diagnostic and statistical manual of mental disorders, Washington, DC, American Psychiatric Association.

Bambico, FR, et al., 2008. The cannabinoid CB1 receptor and the endocannabinoid anandamide: possible antidepressant targets. Expert Opin Ther Targets 12, 1347-66.

Baumann, PS, et al., 2013. Treatment and early intervention in psychosis program (TIPP-Lausanne): Implementation of an early intervention programme for psychosis in Switzerland. Early Interv Psychiatry 7, 322-8.

Belvederi Murri, M, et al., 2015. Is good insight associated with depression among patients with schizophrenia? Systematic review and meta-analysis. Schizophr Res 162, 234-47.

Campos, MS, et al., 2011. Premorbid personality and insight in first-episode psychosis. Schizophr Bull $37,52-60$.

Coentre, $R$, et al., 2017. Depressive symptoms and suicidal behavior after first-episode psychosis: A comprehensive systematic review. Psychiatry research 253, 240-248.

Coldham, EL, et al., 2002. Medication adherence of individuals with a first episode of psychosis. Acta Psychiatr Scand 106, 286-90.

Conus, P, et al., 2007. The First-Episode Psychosis Outcome Study: premorbid and baseline characteristics of an epidemiological cohort of 661 first-episode psychosis patients. Early Interv Psychiatry 1, 191-200.

Drake, RE, et al., 1990. Diagnosis of alcohol use disorders in schizophrenia. Schizophr Bull 16, 57-67. 
Elowe, J, et al., 2017. Much ado about everything: A literature review of insight in first episode psychosis and schizophrenia. European psychiatry : the journal of the Association of European Psychiatrists 39, 73-79.

Fenton, WS, et al., 1997. Determinants of medication compliance in schizophrenia: empirical and clinical findings. Schizophr Bull 23, 637-51.

Gerretsen, P, et al., 2014. The VAGUS insight into psychosis scale--self-report and clinician-rated versions. Psychiatry Res 220, 1084-9.

Grover, S, et al., 2018. Association of internalized stigma and insight in patients with schizophrenia. International Journal of Culture and Mental Health 11, 338-350.

Klaas, HS, et al., 2017. Insight as a social identity process in the evolution of psychosocial functioning in the early phase of psychosis. Psychological medicine $47,718-729$.

Lambert, M, et al., 2005. The impact of substance use disorders on clinical outcome in 643 patients with first-episode psychosis. Acta Psychiatr Scand 112, 141-8.

Lev-Ran, S, et al., 2014. The association between cannabis use and depression: a systematic review and meta-analysis of longitudinal studies. Psychol Med 44, 797-810.

Lysaker, $\mathrm{PH}$, et al., 2007. Toward understanding the insight paradox: internalized stigma moderates the association between insight and social functioning, hope, and self-esteem among people with schizophrenia spectrum disorders. Schizophr Bull 33, 192-9.

Lysaker, PH, et al., 2013. Depression and insight in schizophrenia: comparisons of levels of deficits in social cognition and metacognition and internalized stigma across three profiles. Schizophr Res 148, 18-23.

Misdrahi, D, et al., 2014. Depression in schizophrenia: the influence of the different dimensions of insight. Psychiatry Res 216, 12-6.

Moore, TH, et al., 2007. Cannabis use and risk of psychotic or affective mental health outcomes: a systematic review. Lancet 370, 319-28.

Morosini, PL, et al., 2000. Development, reliability and acceptability of a new version of the DSM-IV Social and Occupational Functioning Assessment Scale (SOFAS) to assess routine social functioning. Acta Psychiatr Scand 101, 323-9.

Myles, H, et al., 2016. Cannabis use in first episode psychosis: Meta-analysis of prevalence, and the time course of initiation and continued use. Aust N Z J Psychiatry 50, 208-19.

Olfson, M, et al., 1999. Assessing clinical predictions of early rehospitalization in schizophrenia. J Nerv Ment Dis 187, 721-9.

Olmos, A, et al., 2018. The efficacy of computerized interventions to reduce cannabis use: $A$ systematic review and meta-analysis. Addict Behav 79, 52-60.

Palmer, EC, et al., 2015. The relationship between cognitive insight and depression in psychosis and schizophrenia: a review and meta-analysis. Schizophr Res 166, 261-8.

Rooke, S, et al., 2013. Effectiveness of a self-guided web-based cannabis treatment program: randomized controlled trial. J Med Internet Res 15, e26.

Saravanan, B, et al., 2010. Outcome of first-episode schizophrenia in India: Iongitudinal study of effect of insight and psychopathology. Br J Psychiatry 196, 454-9.

Schimmelmann, BG, et al., 2012. Prevalence and impact of cannabis use disorders in adolescents with early onset first episode psychosis. Eur Psychiatry 27, 463-9.

Vilaplana, M, et al., 2015. Insight into mental disorders and suicidal behavior: a qualitative and quantitative multimodal investigation. J Clin Psychiatry 76, 303-18.

Yung, AR, et al., 2005. Mapping the onset of psychosis: the Comprehensive Assessment of At-Risk Mental States. Aust N Z J Psychiatry 39, 964-71.

Zammit, S, et al., 2008. Effects of cannabis use on outcomes of psychotic disorders: systematic review. Br J Psychiatry 193, 357-63. 
Table 1. Patients demographic, functional and clinical variables characteristics

\begin{tabular}{|c|c|}
\hline & Total $\mathrm{N}=214$ \\
\hline Age in year, Mean (SD) & $24.2(4.9)$ \\
\hline Sex, \% male $(\mathrm{N})$ & $66.8(143)$ \\
\hline \multicolumn{2}{|l|}{$\mathrm{SES}, \%(\mathrm{~N})$} \\
\hline Low & $16.8(36)$ \\
\hline Intermediate. & $44.9(96)$ \\
\hline High & $38.3(82)$ \\
\hline \multicolumn{2}{|l|}{ Diagnostic, \% (N) } \\
\hline Schizophrenia & $60.7(130)$ \\
\hline Schizophreniform/brief psychotic episode & $11.7(25)$ \\
\hline Schizoaffective disorder & $9.8 / 821)$ \\
\hline Major depression with psychotic features & $2.8(6)$ \\
\hline Bipolar disorder & $6.5(14)$ \\
\hline Others & $8.4(18)$ \\
\hline \multicolumn{2}{|l|}{ Suicide attempts during program, \% (N) } \\
\hline None & $95.8(205)$ \\
\hline One attempt & $3.3(7)$ \\
\hline Several attempts & $1.0(2)$ \\
\hline \multicolumn{2}{|l|}{ SOFAS, Mean (SD) } \\
\hline Baseline & \\
\hline 2 months & $38.2(15.6)$ \\
\hline 12 months & $\begin{array}{l}49.8(15.3) \\
57.5(16.2)\end{array}$ \\
\hline 18 months & $58.5(15.5)$ \\
\hline 24 months & $58.0(16.0)$ \\
\hline 36 months & $58.7(16.3)$ \\
\hline \multicolumn{2}{|l|}{$\begin{array}{l}\text { PANSS Positive, Mean } \\
2 \text { months }\end{array}$} \\
\hline 12 months & $13.2(4.6)$ \\
\hline 18 months & $11.8(4.3)$ \\
\hline 24 months & $12.1(4.2)$ \\
\hline 36 months & $12.0(4.9)$ \\
\hline \multirow{2}{*}{\multicolumn{2}{|c|}{$\begin{array}{l}\text { PANSS Negative, Mean (SD) } \\
2 \text { months }\end{array}$}} \\
\hline & \\
\hline 12 months & $15.4(6.2)$ \\
\hline 18 months & $14.5(5.6)$ \\
\hline 24 months & $15.0(5.9)$ \\
\hline 36 months & $13.7(5.1)$ \\
\hline \multirow{2}{*}{\multicolumn{2}{|c|}{$\begin{array}{l}\text { MADRS, Mean (SD) } \\
2 \text { months }\end{array}$}} \\
\hline & \\
\hline 12 months & $14.8(9.1)$ \\
\hline 18 months & $11.9(8.9)$ \\
\hline 24 months & $12.8(9.4)$ \\
\hline 36 months & $11.1(8.2)$ \\
\hline \multicolumn{2}{|l|}{ CMRS, Mean (SD) } \\
\hline Baseline & $17(1.0)$ \\
\hline 2 months & $\begin{array}{l}.7(1.0) \\
1.5(0.9)\end{array}$ \\
\hline 12 months & $1.5(0.9)$ \\
\hline 18 months & $1.4(0.7)$ \\
\hline 24 months & $1.5(0.9)$ \\
\hline 36 months & $1.4(0.8)$ \\
\hline \multicolumn{2}{|l|}{$\begin{array}{l}\text { Medication adherence }{ }^{\mathrm{a}}, \% \\
2 \text { months }\end{array}$} \\
\hline 12 months & $63.1(135)$ \\
\hline 18 months & $64.0(119)$ \\
\hline 24 months & $66.7(118)$ \\
\hline 36 months & $70.6(113)$ \\
\hline & $75.6(118)$ \\
\hline \multicolumn{2}{|l|}{ Insight $^{\mathrm{b}}, \%(\mathrm{~N})$} \\
\hline Baseline & \\
\hline 2 months & $\begin{array}{l}18.9(39) \\
30.4(65)\end{array}$ \\
\hline 12 months & $\begin{array}{l}50.4(03) \\
45.9(84)\end{array}$ \\
\hline 18 months & $48.9(85)$ \\
\hline 24 months & $50.6(82)$ \\
\hline 36 months & $54.5(84)$ \\
\hline
\end{tabular}

PANSS $=$ Positive and Negative Syndrome Scale. MADRS = Montgomery Asberg Depression Rating Scale.

$\mathrm{GAF}=$ Global Assessment of Functioning Scale $. \mathrm{CMRS}=$ Case Manager Rating Scale. Medication adherence ${ }^{\mathrm{a}}=$ complete medication adherence. Insight ${ }^{\mathrm{b}}=$ complete insight. 
Figure 1. Relationship between Insight, Medication adherence and Cannabis use at the beginning of the program and depression one year later.

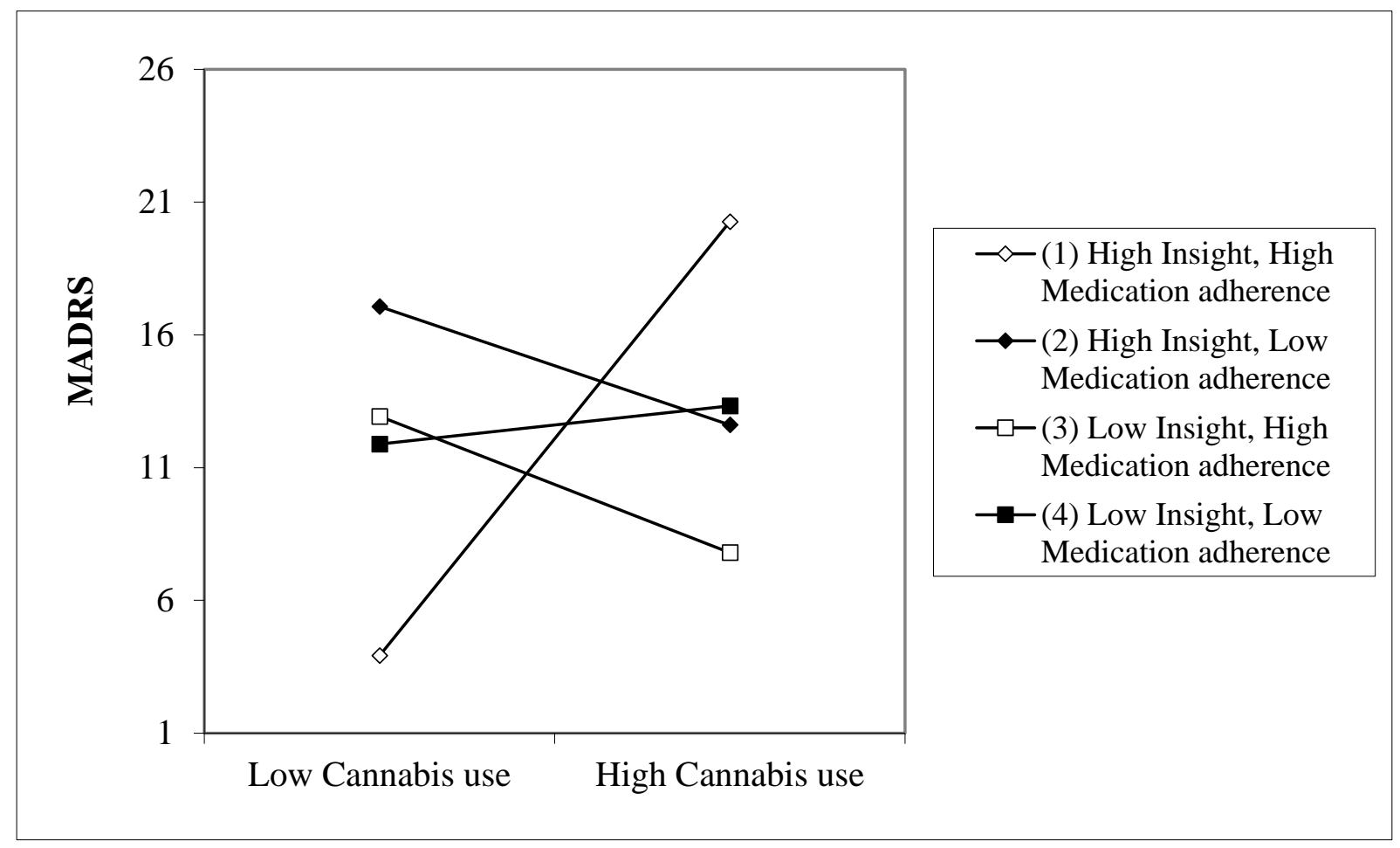

Figure 2. Relationship between Insight, Medication adherence and Cannabis use at the beginning of the program and positive symptomatology (PANSS) 24 months later.

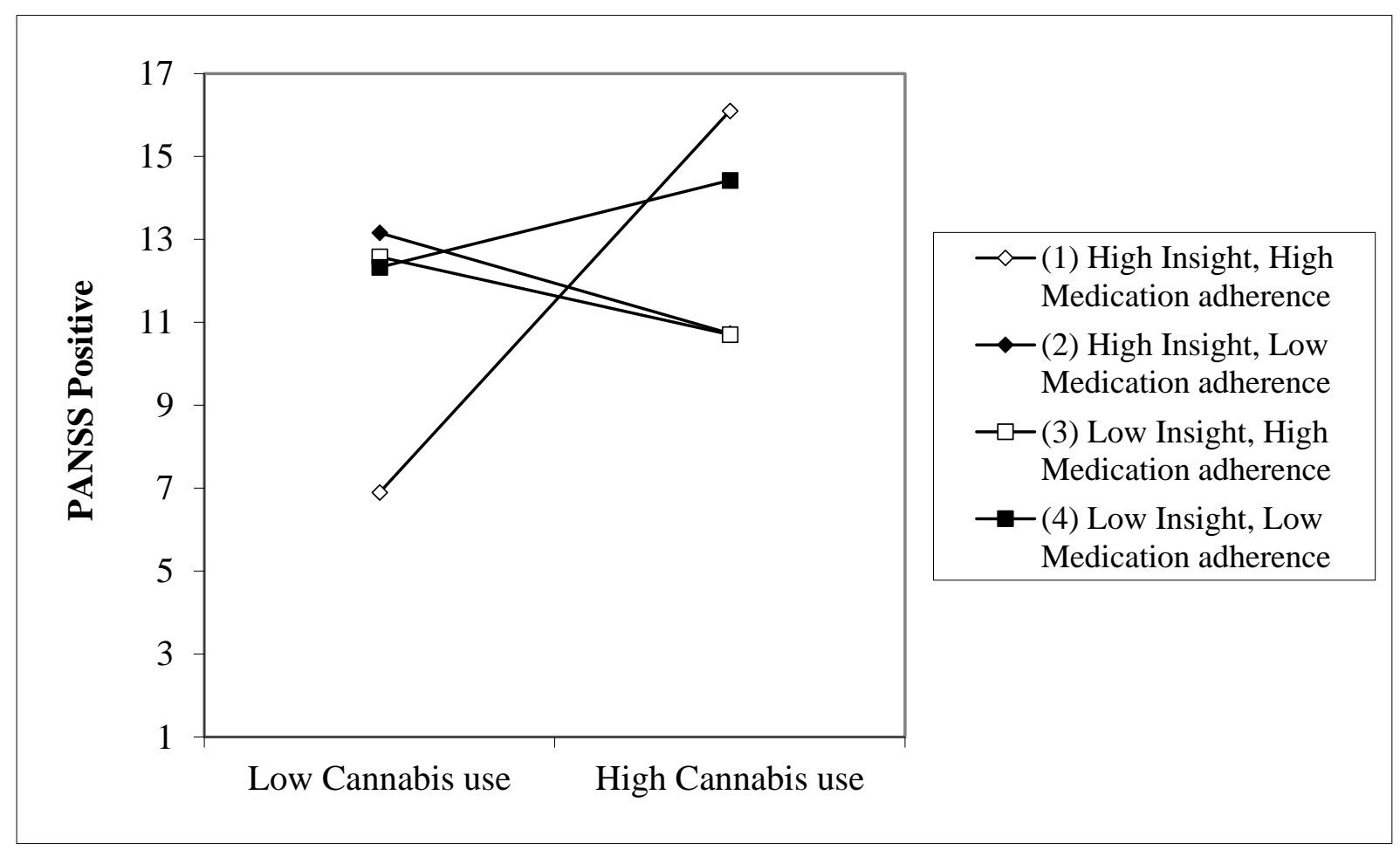

